Chemical Physics 127 (1988) 305-312

North-Holland, Amsterdam

\title{
ESR STUDIES OF METALLOCENE INTERCALATED $\mathrm{Cd}_{2} \mathbf{P}_{2} \mathrm{~S}_{6}$
}

\author{
E. LIFSHITZ, D.A. CLEARY and A.H. FRANCIS \\ Department of Chemistry, University of Michigan, Ann Arbor, MI 48109-1055, USA
}

Received 22 June 1988

\begin{abstract}
$\mathrm{Cd}_{2} \mathrm{P}_{2} \mathrm{~S}_{6}$ exhibits a layered crystal structure that may be intercalated with metallocenes. The ESR spectra of $\mathrm{Cd}_{2} \mathrm{P}_{2} \mathrm{~S}_{6}$ single crystals intercalated with cobaltocene, nickelocene and chromocene have been examined over the temperature range $280-4.2 \mathrm{~K}$. The spectra indicate that both oxidized and unoxidized metallocene is present in the van der Waals gap of the layered host lattice. Examination of the angle dependence of the ESR spectra establishes the orientation of neutral cobaltocene and nickelocenium cation with the $\mathrm{C}_{5}$ molecular symmetry axis parallel to the $C^{*}$ axis of the host lattice. The orientation of chromocenium cation is rotated $90^{\circ}$ to place $C_{5}$ perpendicular to $C^{*}$.
\end{abstract}

\section{Introduction}

Transition metal chalcogenophosphates $\left(\mathrm{M}_{2} \mathrm{P}_{2} \mathrm{X}_{6}\right.$, $\mathrm{M}=$ transition metal, $\mathrm{X}=\mathrm{S}, \mathrm{Se}$ ) crystallize in a lamellar arrangement, similar in form to the transition metal dichalcogenides $\mathrm{MX}_{2}$, in which metal ions and $\mathrm{P}_{2}$ atom pairs are sandwiched between planes of hexagonally packed chalcogen atoms to form a three atom thick layer. The macroscopic structure is obtained by stacking the layers along the $C^{*}$ axis as illustrated in fig. $1[1,2]$. Adjacent layers are weakly bonded by van der Waals interactions and the space between the layers is referred to as the van der Waals gap (VWG). Because of the weak interlayer interactions, it is possible to intercalate small ions, organic molecules, and

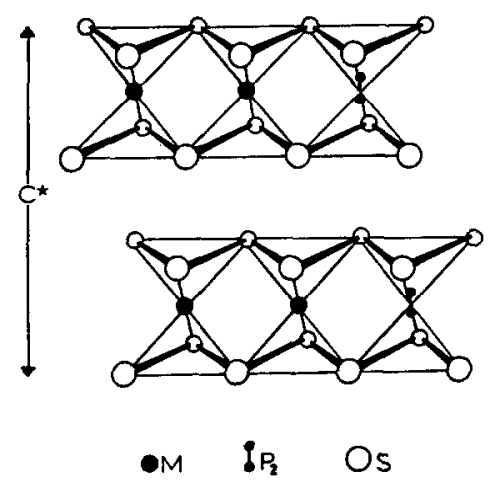

Fig. 1. The layered structure of $\mathrm{MPX}_{3}$ materials. organometallic complexes into the VWG region.

The metallocene intercalation compounds of $\mathrm{MX}_{2}$ lattices have been studied for about ten years [3-11]. Metallocene compounds of $\mathrm{M}_{2} \mathrm{P}_{2} \mathrm{X}_{6}$ have also been investigated by a number of researchers [12-23]. Metallocenes will intercalate many of the $\mathbf{M X}_{2}$ and $\mathrm{M}_{2} \mathrm{P}_{2} \mathrm{X}_{6}$ hosts, but the intercalation mechanism and the structure of the intercalation compounds that are formed is variable. In much of the previous work, both the structure and the oxidation state of the intercalated metallocene has been of interest.

\subsection{Structure}

Attempts have been made to infer the structure of metallocene intercalation compounds from the lattice basal expansion. For example, chromocene $\left(\mathrm{Cr}(\mathrm{cp})_{2}, \mathrm{cp}=\right.$ cyclopentadienyl $)$ and cobaltocene $\left[\mathrm{Co}(\mathrm{cp})_{2}\right]$ have been intercalated into $\mathrm{TaS}_{2}$. The size of the metallocene and the magnitude of the interplanar lattice expansion suggested an orientation of the metallocene with the five-fold molecular symmetry axis $\left(\mathrm{C}_{5}\right)$ perpendicular to the stacking axis of the host lattice [3]. The notation for this orientation, $\mathrm{C}_{5} \perp C^{*}$, is used throughout the text and the orientation is illustrated in fig. 2.

It has been observed [4] that the unsubstituted metallocenes have approximately equal dimensions along their principal axes and it is not possible to in- 


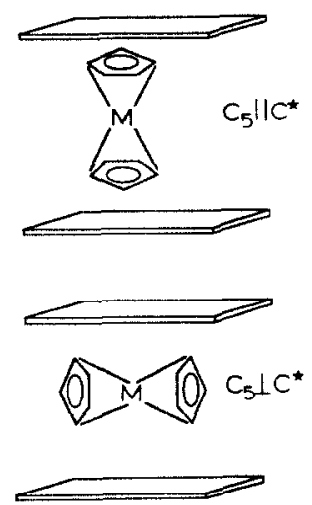

Fig. 2. Possible equilibrium orientations of $M(c p)_{2}$ in the VWG.

fer the molecular orientation from the basal plane expansion. This point notwithstanding, studies by Silbernagel [5] of the wide-line NMR spectrum of $\mathrm{Co}(\mathrm{cp})_{2}$ in $\mathrm{TaS}_{2}$ gave results consistent with a $\mathrm{C}_{5} \perp C^{*}$ equilibrium orientation. The observation that the lattice expansion increases as the metallocene ring size increases also suggests a $C_{5} \perp C^{*}$ orientation [4]. However, the increased basal expansion observed when $\mathrm{TaS}_{2}$ is intercalated with substituted metallocenes, suggests a $C_{5} \| C^{*}$ structure [4].

Fewer structural studies of metallocene intercalated $\mathrm{M}_{2} \mathrm{P}_{2} \mathrm{~S}_{6}$ lattices have been reported. The relative intensity of infrared bands associated with inplane and out-of-plane ring deformations of intercalated $\mathrm{Co}(\mathrm{cp})_{2}$ suggests an orientation of the intercalate with $\mathrm{C}_{5} \perp \boldsymbol{C}^{*}$ [12]. However, ESR studies of Co(cp) 2 intercalated $\mathrm{Cd}_{2} \mathrm{P}_{2} \mathrm{~S}_{6}$ indicated a $\mathrm{C}_{5} \| C^{*}$ equilibrium orientation [13].

\subsection{Metallocene oxidation}

Determination of the oxidation state of the intercalated metallocene is important for an understanding of the intercalation reaction mechanism. It is frequently observed that the metallocene is present as a cation in both $\mathrm{MX}_{2}$ and $\mathrm{M}_{2} \mathrm{P}_{2} \mathrm{X}_{6}$ lattices. The evidence for this conclusion is obtained from examination of the optical spectrum [14,15], the magnetic susceptibility $[14,16]$ and the vibrational spectra [17,18] of the intercalated lattices. For example, cobaltocene and chromocene are fully ionized when intercalated into $\mathrm{TaS}$, resulting in a diamagnetic complex for cobaltocene and a paramagnetic com- plex for chromocene [6]. Therefore, the process of intercalation appears to involve the donation of an electron to the host lattice. The transferred electron does not contribute to the paramagnetism of the material, suggesting that it is spin-paired [141. Vibrational spectra of $\mathrm{Cd}_{2} \mathrm{P}_{2} \mathrm{~S}_{6}$ intercalated with either neutral cobaltocene or cobaltocenium ion exhibited bands belonging to the $\mathrm{Co}(\mathrm{cp})_{2}^{+}$species and samples prepared by both methods were found to be diamagnetic at $293 \mathrm{~K}[12,14] . \mathrm{Co}(\mathrm{cp})_{2}^{+}$intercalates $\mathrm{M}_{2} \mathrm{P}_{2} \mathrm{~S}_{6}$ lattices with $\mathrm{M}=\mathrm{Mn}, \mathrm{Zn}$ and $\mathrm{Cd}$ to give compounds identical to those obtained starting from the neutral guest molecules $[12,14]$. This implies a redox reaction mechanism for the intercalation of neutral metallocene, involving oxidation of the metallocene and transfer of an electron to the host lattice. A recent Mössbaucr study of the intercalation of ferrocene into $\mathrm{Cd}_{2} \mathrm{P}_{2} \mathrm{~S}_{6}$ indicated the presence of the ferrocene cation in several distinct sites and with a variety of oxidation states [19].

Alternatively, the metallocene cation may be intercalated by an ion exchange mechanism in which intralamellar $\mathrm{M}^{2+}$ cation is displaced from the lattice to achieve charge compensation [20]. This process leads to interlayer metallocene cation guests and intralayer transition metal cation vacancies.

This paper reports the results of ESR studies of metallocenes $\left(\mathrm{M}(\mathrm{cp})_{2}, \mathrm{M}=\mathrm{Ni}, \mathrm{Cr}, \mathrm{Co}\right)$ intercalated into $\mathrm{Cd}_{2} \mathrm{P}_{2} \mathrm{~S}_{6}$. ESR spectroscopy is used to examine the oxidation state and equilibrium structure of the intercalated metallocene in the host lattice.

\section{Experimental}

$\mathrm{Cd}_{2} \mathrm{P}_{2} \mathrm{~S}_{6}$ was prepared from a stoichiometric mixture of the elements following procedures reported prcviously [24]. Large, singlc crystal samples can be obtained by this procedure. The material is typically slightly metal deficient and sulfur rich. Crystal samples were obtained as nearly colorless platelets of approximate dimensions $3 \times 3 \times 0.1 \mathrm{~mm}^{3}$. Elemental analysis of the product gave: $\mathrm{Cd}$ (calc. $46.92 \%$, obs. $44.9 \%$ ), P (calc. $12.93 \%$, obs. $12.92 \%$ ) and S (calc. $40.15 \%$, obs. $42.18 \%$ ). The basal spacing was found to be $6.42 \AA$.

In order to obtain uniform intercalation of large, single crystals, the metallocene intercalation reaction 
was carried out in two steps: first, single crystals of $\mathrm{Cd}_{2} \mathrm{P}_{2} \mathrm{~S}_{6}$ were intercalated with pyridine by immersion in pyridine at room temperature for a period of 7 days. Chemical analysis of the pyridine (py) intercalated $\mathrm{Cd}_{2} \mathrm{P}_{2} \mathrm{~S}_{6}$ indicated that the chemical formula was $\mathrm{Cd}_{2} \mathrm{P}_{2} \mathrm{~S}_{6}$ (py) $)_{0.5}$. Second, single crystals of the pyridine intercalation compound were placed in a toluene solution of the metallocene and heated to $135^{\circ} \mathrm{C}$ for $3-5$ days. The toluene solvent used had been distilled and stored over molecular sieve and all manipulations were conducted in a dry nitrogen atmosphere with $\mathrm{O}_{2}+\mathrm{H}_{2} \mathrm{O}<50$ ppm.

Cobaltocene intercalated crystals were nearly opaque, appearing red in transmitted light. Chemical analysis of the intercalated lattice yielded $34.99 \% \mathrm{Cd}$, $7.23 \% \mathrm{Co}, 16.62 \% \mathrm{C}, 1.58 \% \mathrm{H},<0.1 \% \mathrm{~N}$, consistent with the approximate empirical formula $\mathrm{Cd}_{2} \mathrm{P}_{2} \mathrm{~S}_{6}\left[\mathrm{Co}(\mathrm{cp})_{2}\right]_{0.79}$. The hydrogen analysis suggests that some water was also present and the nitrogen analysis indicated that only a trace amount of pyridine was retained.

The nickelocene intercalation compound had a black, lustrous appearance. Chemical analysis gave: $33.88 \% \mathrm{Cd}, 10.38 \% \mathrm{P}, 35.29 \% \mathrm{~S}, 4.93 \% \mathrm{Ni}, 13.53 \%$ $\mathrm{C}, 1.38 \% \mathrm{H}, 0.096 \% \mathrm{~N}$, from which the following molecular formula was derived: $\mathrm{Cd}_{1.80} \mathrm{P}_{2} \mathrm{~S}_{6.57^{-}}$ $\left[\mathrm{Ni}(\mathrm{cp})_{2}\right]_{0.50}$. The basal spacing was found to be 12.1 $\AA$.

Crystals of the chromocene intercalated lattice were lustrous, with a deep red color. Chemical analysis gave: $35.3 \% \mathrm{Cd}, 11.2 \% \mathrm{P}, 34.5 \% \mathrm{~S}, 4.86 \% \mathrm{Cr}$, $11.2 \% \mathrm{C}, 1.4 \% \mathrm{H}$ and $<0.1 \% \mathrm{~N}$, from which the following molecular formula was derived: $\mathrm{Cd}_{1.74} \mathrm{P}_{2} \mathrm{~S}_{6}\left[\mathrm{Cr}(\mathrm{cp})_{2}\right]_{0.52}$. The basal spacing from single crystal X-ray diffraction was $12.07 \AA$.

$X$-band ESR spectra were recorded with a Bruker ER 200E-SRC spectrometer equipped with a $\mathrm{TM}_{110}$ cylindrical cavity. The sample crystals used were of approximate dimensions $3 \mathrm{~mm} \times 3 \mathrm{~mm} \times 0.1 \mathrm{~mm}$. ESR spectra were obtained with the external magnetic field directed either parallel $\left(\boldsymbol{H} \| \boldsymbol{C}^{*}\right)$ or perpendicular $\left(H \perp C^{*}\right)$ to the crystallographic stacking axis $C^{*}$. Low-temperature spectra were recorded using continuous-flow, liquid-nitrogen and liquid-helium cooled ESR cryostats.

\section{Results}

$\mathrm{X}$-band ESR spectra of the cobaltocene intercalated $\mathrm{Cd}_{2} \mathrm{P}_{2} \mathrm{~S}_{6}$ obtained at $113 \mathrm{~K}$ with $\boldsymbol{H} \| \boldsymbol{C}^{*}$ and $\boldsymbol{H} \perp \boldsymbol{C}^{*}$ are illustrated in fig. 3. The $\boldsymbol{H} \| \boldsymbol{C}^{*}$ spectrum consists of the eight nuclear hyperfine components of a single electron spin transition. When the sample is oriented with $\boldsymbol{H} \perp \boldsymbol{C}^{*}$, no hyperfine structure is resolved and the resonance signal is an order of magnitude weaker. The spectrum is strongly temperature dependent and no signal could be observed above about $120 \mathrm{~K}$. The ESR spectra of $\mathrm{Ni}(\mathrm{cp})_{2}$ intercalated $\mathrm{Cd}_{2} \mathrm{P}_{2} \mathrm{~S}_{6}$ obtained with $\boldsymbol{H} \| \boldsymbol{C}^{*}$ and $\boldsymbol{H} \perp \boldsymbol{C}^{*}$ are shown in fig. 4 . The spectra are strongly temperature dependent.

The room temperature ESR spectrum of chromocene intercalated $\mathrm{Cd}_{2} \mathrm{P}_{2} \mathrm{~S}_{6}$ consists of a broad $(\Delta H=1370 \mathrm{G})$, angle-independent resonance at approximately $3333 \mathrm{G}$. As the temperature of the sample is decreased, the resonance signal intensity decreases and the linewidth narrows. Additionally, a narrow, angle dependent resonance appears at 1667 G. The spectra obtained at $77 \mathrm{~K}$ with $\boldsymbol{H} \| \boldsymbol{C}^{*}$ and $H \perp C^{*}$ are shown in fig. 5 . The $1667 \mathrm{G}$ resonance is usually accompanied by one or two satellite resonances whose relative intensity varies with sample.

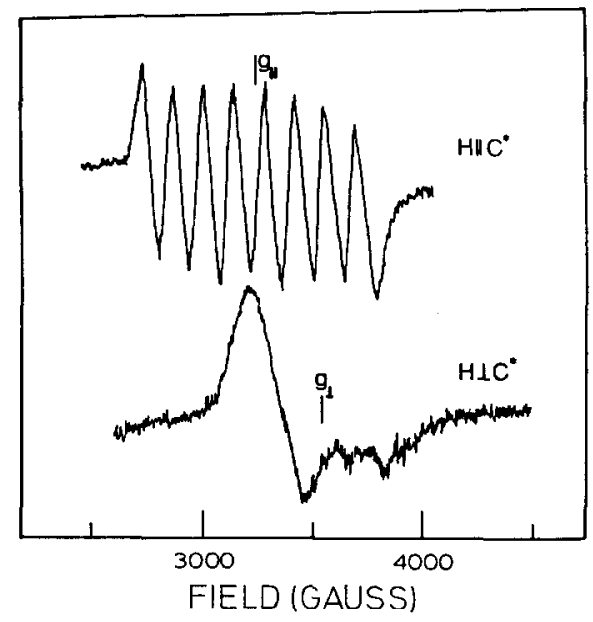

Fig. 3. ESR spectra of cobaltocene intercalated $\mathrm{Cd}_{2} \mathrm{P}_{2} \mathrm{~S}_{6}$ obtained with $\boldsymbol{H} \| \boldsymbol{C}^{*}$ and $\boldsymbol{H} \perp \boldsymbol{C}^{*}$. 


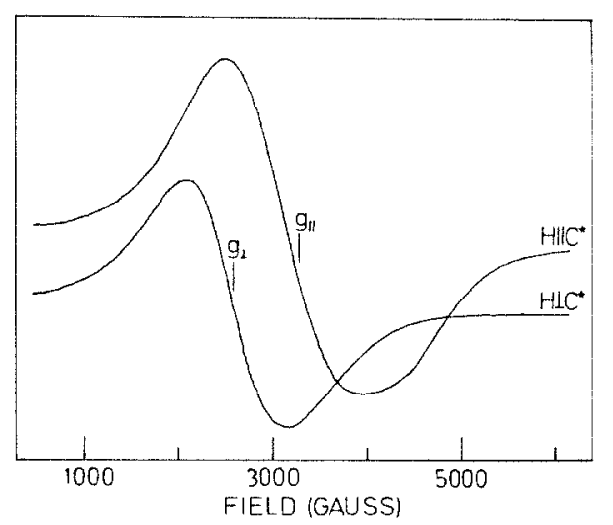

Fig. 4. The ESR spectrum of $\mathrm{Ni}(\mathrm{cp})_{2}$ intercalated $\mathrm{Cd}_{2} \mathrm{P}_{2} \mathrm{~S}_{6}$ obtaned with $\boldsymbol{H} \| \boldsymbol{C}^{*}$ and $\boldsymbol{H} \perp \boldsymbol{C}^{*}$.

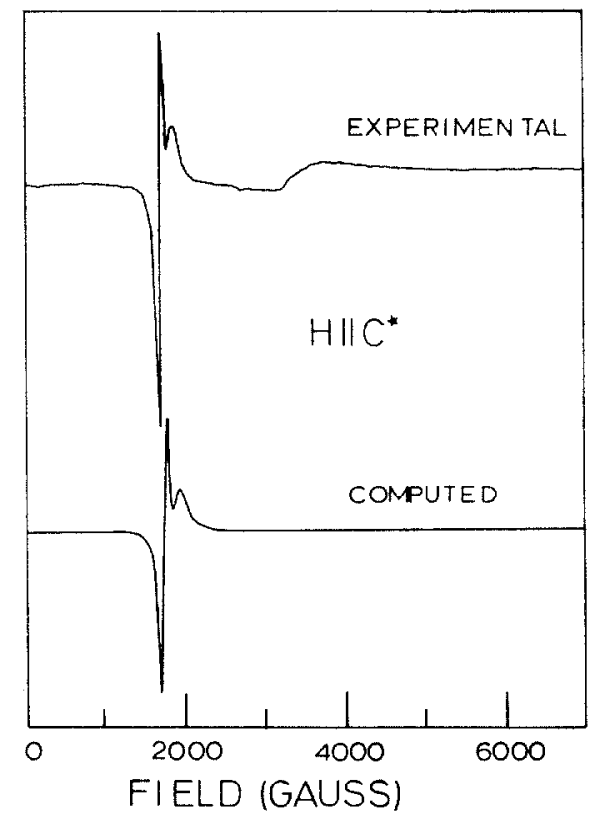

Fig. 5. (Top) ESR spectrum of $\mathrm{Cr}(\mathrm{cp})_{2}$ intercalated $\mathrm{Cd}_{2} \mathrm{P}_{2} \mathrm{~S}_{6}$ obtained with $\boldsymbol{H} \| \boldsymbol{C}^{*}$. (Bottom) computed spectrum.

\section{Discussion}

Most of the metallocenes are paramagnetic, either as neutral or as cations. The ESR spectra have been investigated in a variety of matrices and the results are summarized in reviews by Ammeter [25] and Warren [26]. In most cases the ESR spectra are reasonably well understood and can be used to identify the oxidation state and the structure of the intercalated metallocene in the layered $\mathrm{Cd}_{2} \mathrm{P}_{2} \mathrm{~S}_{6}$ host lattice.

Cobaltocene, nickelocene and chromocene have $\mathrm{D}_{5 \mathrm{~d}}$ axial symmetry and the molecular orbital description may be constructed from symmetry adapted ligand $\pi$ orbitals and transition metal d orbitals. The low-energy orbitals are predominantly ligand in character and are fully occupied; the high-energy orbitals are partially occupied and have predominantly $3 \mathrm{~d}$ metal character. Therefore, the ESR spectra may be qualitatively discussed in terms of the splitting of $3 \mathrm{~d}$ orbitals in the axial field of the cyclopentadienyl ligands. In an axial field the transition metal $3 \mathrm{~d}$ orbitals are split into three sets: $\sigma\left(\mathrm{d}_{z 2}\right), \pi\left(\mathrm{d}_{x z}, \mathrm{~d}_{y z}\right)$ and $\delta\left(\mathrm{d}_{x^{2}-y^{2}}, \mathrm{~d}_{x y}\right)$. Usually the one-electron core energies follow the order $\delta<\sigma \ll \pi$. The $\delta$ level is strongly bonding, the $\sigma$ level less so and the $\pi$ level strongly antibonding. The axial field splitting of the $3 \mathrm{~d}$ transition metal orbitals is illustrated in fig. 6 .

The neutral metallocene electronic configurations are: cobaltocene, $d^{7}\left(\delta^{4} \sigma^{2} \pi^{1}\right)$; nickelocene, $d^{8}\left(\delta^{4} \sigma^{2} \pi^{2}\right)$ and chromocene, $\mathrm{d}^{4}\left(\delta^{3} \sigma^{1} \pi^{0}\right)$. The paramagnetism of cobaltocene, nickelocene, chromocene and their monopositive cations, may be described by the following spin-Hamiltonian:

$$
\begin{aligned}
\mathscr{H} & =\beta\left(\boldsymbol{L}+\mathbf{g}_{\mathrm{c}} \boldsymbol{S}\right) \cdot \boldsymbol{H}+D\left(S_{z}^{2}-\frac{1}{3} S^{2}\right)-E\left(S_{x}^{2}-S_{y}^{2}\right) \\
& +h\left[A_{\|} S_{z} I_{z}+A_{\perp}\left(S_{x} I_{x}+S_{y} I_{y}\right)\right]+\lambda \boldsymbol{L} \cdot \boldsymbol{S} .
\end{aligned}
$$

In eq. (1), $\beta$ is the Bohr magneton, $\boldsymbol{H}$ is the external magnetic field, and $\mathbf{g}_{\mathrm{e}}$ is the free-electron $g$-tensor. $D$ and $E$ are the axial and orthorhombic crystal field parameter, respectively. $A_{\|}$and $A_{\perp}$ are the components

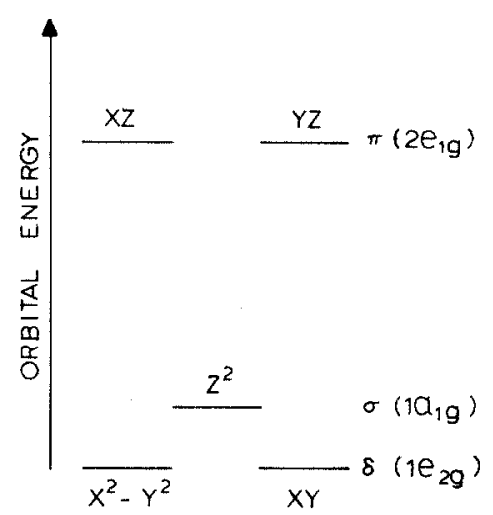

Fig. 6. The splitting of $3 \mathrm{~d}$ orbitals in $\mathrm{D}_{5 \mathrm{~d}}$ axial ligand field. 
of an axial nuclear hyperfine coupling tensor. $\boldsymbol{L}$ and $S$ are the electronic orbital and spin angular momentum operators and $I$ is the nuclear spin operator. The axial symmetry of the free metallocene is reduced upon intercalation by crystal field and intermolecular interactions within the VWG. Under these circumstances, the paramagnetism is adequately described by eq. (1), which is appropriate for orthorhombic or lower symmetry. The $\mathrm{C}_{5}$ axial symmetry of the free metallocenes requires the addition of higher-order terms to the Hamiltonian.

\subsection{Cobaltocene intercalation compounds of $\mathrm{Cd}_{2} \mathrm{P}_{2} \mathrm{~S}_{6}$}

The $\mathrm{d}^{7}\left(\delta^{4} \sigma^{2} \pi^{1}\right)$ configuration of $\mathrm{Co}(\mathrm{cp})_{2}$ places a single unpaired electron in the antibonding $\pi$ orbital. The ESR spectrum of the neutral $\mathrm{Co}(\mathrm{cp})_{2}$ intercalate may be analyzed with the spin-Hamiltonian of eq. (1). Since $S=1 / 2$, the terms in $S^{2}$ are zero and, assuming the electron spin to be quantized along the external magnetic field direction, eq. (1) can be rewritten as:

$\mathscr{H}=\beta g_{\mathrm{eff}} S_{z} H+h\left[A_{\|} S_{z} I_{z}+A_{\perp}\left(S_{x} I_{x}+S_{y} I_{y}\right)\right]$.

Here, $g_{\text {eff }}$ is the effective $g$-tensor given by:

$g_{\text {eff }}^{2}(\theta)=g_{\text {月 }}^{2} \cos ^{2} \theta+g_{\perp}^{2} \sin ^{2} \theta$

and $\theta$ is the angle between $\boldsymbol{H}$ and $\mathrm{C}_{5}$. The angular dependence of the resonance field $H_{\mathrm{r}}$ is given by:

$H_{\mathrm{r}}(\theta)=\frac{h \nu}{\beta g_{\mathrm{eff}}(\theta)}$.

The anisotropy in $g$ shifts the ESR spectrum obtained with $\theta=0^{\circ}\left(\boldsymbol{H} \| \mathrm{C}_{5}\right)$ to low-field relative to that obtained with $\theta=90^{\circ}\left(\boldsymbol{H} \perp \mathrm{C}_{5}\right)$. The cobalt nuclear spin $(I=7 / 2)$ results in the splitting of the single $S=1 / 2$ electron-spin transition into a nuclear hyperfine octet. The hyperfine structure is expected to be well resolved in the $g_{\|}$spectrum, and poorly resolved in the $g_{\perp}$ spectrum due to the large anisotropy $\left(A_{\|} \gg A_{\perp}\right)$ of the nuclear hyperfine coupling tensor [27].

According to the above discussion, the well-resolved, low-field octet (fig. 3) obtained with $\boldsymbol{H} \| \boldsymbol{C}^{*}$, arises from neutral $\mathrm{Co}(\mathrm{cp})_{2}$ with $\mathrm{C}_{5} \| \boldsymbol{H}$. Therefore, the $\mathrm{Co}(\mathrm{cp})_{2}$ orientation is $\mathrm{C}_{5} \| C^{*}$. The $\boldsymbol{H} \perp C^{*}$ spectrum in fig. 3 corresponds to that expected for orien- tation of $\mathrm{Co}(\mathrm{cp})_{2}$ with $\mathrm{C}_{5} \perp \boldsymbol{H}$. This result requires $\mathrm{C}_{5} \| C^{*}$ and is consistent with the interpretation of the $\boldsymbol{H} \| C^{*}$ spectrum. Analysis of the spectra yields $g_{\|}=2.00$ and $g_{\perp}=1.96$. These values are considerably greater than observed in a variety of other crystal lattices and rare gas matrices [20]. The presence of unquenched orbital angular momentum causes a deviation of the $g$ tensor from its free-electron value and introduces anisotropy in both the electronic Zeeman and nuclear hyperfine interactions. Ammeter and Swalen [27] have computed $g_{\mid}$and $g_{\perp}$ from a simple model in which the molecular orbital of the single unpaired electron is described by a linear combination of cobalt $\mathrm{d}_{x z}$ and $\mathrm{d}_{y z}$ atomic orbitals with ligand orbitals of appropriate symmetry. From these orbital basis functions, the following expressions may be obtained from eq. (2):

$g_{\|}=g_{\mathrm{e}}-2 k_{\|} / \sqrt{1+y^{2}}$,

$g_{\perp}=g_{\mathrm{e}} / \sqrt{1+y^{2}}$,

Here $0<k_{\|}<1$ is the orbital angular momentum reduction factor, and $y=\zeta / 2 \delta$. $\zeta$ is the molecular spinorbit coupling constant and $\delta$ is the static ligand field orthorhombic distortion. As the covalent interaction between the ligands and transition metal $3 \mathrm{~d}$ orbitals increases, the value of $k_{\|}$approaches zero. The $g$-value anisotropy of the metallocenes arises primarily from the static orthorhombic distortion parameter, which is sensitive to the local environment of the metallocene. In general, as the axial symmetry of the metallocene is decreased, both $g_{\|}$and $g_{\perp}$ increase toward the value $g_{\mathrm{e}}=2$. The relatively large values obtained for $g_{\|}$and $g_{\perp}$ indicate a decrease in molecular axial symmetry within the VWG. This may occur either as a result of intercalate-lattice or intermolecular interactions within the VWG.

Cobaltocene cation has a $d^{6}\left(\delta^{4} \sigma^{2} \pi^{0}\right)$ configuration and is not paramagnetic. Therefore, cobaltocenium cation could be present in the VWG and not be observed in the ESR spectrum. Previous studies of intercalated $\mathbf{M}_{2} \mathbf{P}_{2} \mathbf{S}_{6}$ lattices intercalated with cobaltocene have concluded that the species present in the VWG is predominately the cation with a $\mathrm{C}_{5} \perp C^{*}$ orientation [12]. Recent studies of the cobaltocene intercalation compound of layered $\mathrm{SnS}_{2}$ have concluded that both neutral cobaltocene and cobaltocenium ion are present [28]. 


\subsection{Nickelocene intercalation compounds of $\mathrm{Cd}_{2} P_{2} S_{6}$}

The $\mathrm{d}^{8}\left(\delta^{4} \sigma^{2} \pi^{2}\right)$ configuration of $\mathrm{Ni}^{2+}$ in neutral nickelocene yields two unpaired spins. However, nickelocene does not produce an ESR signal because of the very large axial ligand field splitting [25]. Nickelocenium cation $\mathrm{d}^{7}\left(\delta^{4} \sigma^{2} \pi^{1}\right)$ is isoelectric with neutral cobaltocene and the observation of a strong ESR resonance from $\mathrm{Ni}(\mathrm{cp})_{2}$ intercalated $\mathrm{Cd}_{2} \mathrm{P}_{2} \mathrm{~S}_{6}$ (fig. 4) suggests the presence of nickelocenium cation in the VWG.

The appropriate spin-Hamiltonian for nickelocenium ion is given in eq. (2). The angle dependence of the resonance field (eqs. (3) and (6)) is shown in fig. 7 and indicates a preferred alignment of $\mathrm{C}_{5}$ within the VWG.

If a $\mathrm{C}_{5} \perp \mathrm{C}^{*}$ orientation with random orientation of $\mathrm{C}_{5}$ axes parallel to the basal plane is assumed, then as the crystal is rotated about $\boldsymbol{C}^{*} \perp \boldsymbol{H}$ an angle-independent, two-dimensional ESR powder spectrum should be observed (with a feature at $g_{\perp}$ and $g_{\|}$). Rotation about any axis perpendicular to $\mathrm{C}_{5}$ would produce a strongly angle-dependent spectrum, with an intense $g_{\perp}$ spectrum being obtained when $\boldsymbol{H} \| \boldsymbol{C}^{*}$ and

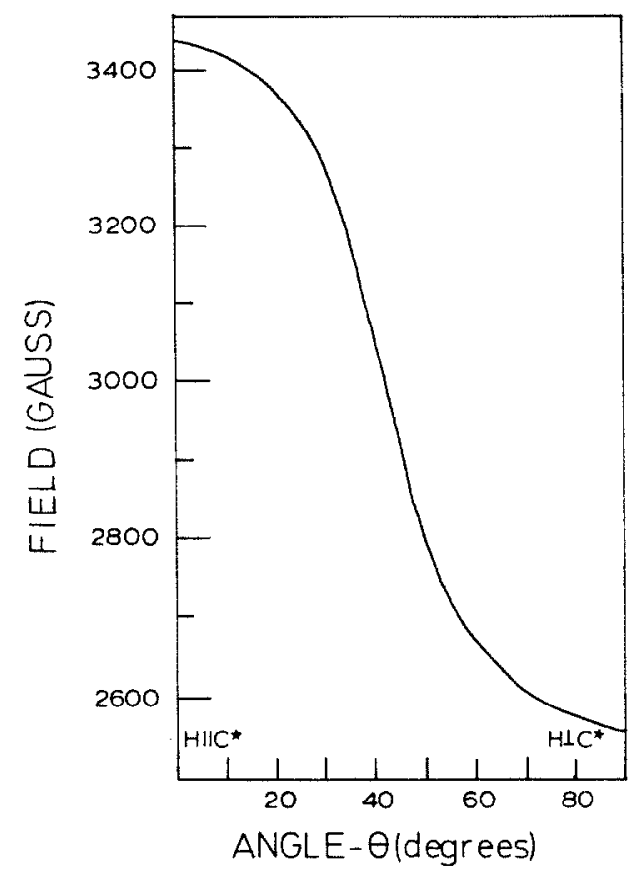

Fig. 7. The angle dependence of $\mathrm{Ni}(\mathrm{cp})_{2}^{+}$resonance in $\mathrm{Cd}_{2} \mathrm{P}_{2} \mathrm{~S}_{6}$. the two-dimensional powder spectrum being obtained with $\boldsymbol{H} \perp \boldsymbol{C}^{*}$.

If a $C_{5} \| C^{*}$ orientation is assumed, rotation about $C^{*} \perp \boldsymbol{H}$ will generate an angle-independent $g_{\perp}$ spectrum. Rotation about any axis perpendicular to $C^{*}$ will produce a strongly angle dependent spectrum varying between $g_{\|}$when $\boldsymbol{H} \| \boldsymbol{C}^{*}$ and $g_{\perp}$ when $\boldsymbol{H} \perp \boldsymbol{C}^{*}$. The ESR spectra are consistent with the $\mathrm{C}_{5} \| C^{*}$ model; the same orientation determined for the isoelectronic metallocene, $\mathrm{Co}(\mathrm{cp})_{2}$ in $\mathrm{Cd}_{2} \mathrm{P}_{2} \mathrm{~S}_{6}$.

\subsection{Chromocene intercalation of $\mathrm{Cd}_{2} P_{2} S_{6}$}

The ground state of chromocene is ${ }^{3} E_{2 g} \mathrm{~d}^{4}\left(\delta^{3} \sigma^{6} \pi^{0}\right)$ and the axial field splitting parameter $(D)$ is calculated to be $-37.2 \mathrm{~cm}^{-1}$ [29]. The allowed X-band ESR transitions of neutral chromocene would occur at very high field $(>10 \mathrm{kG})$ and the spectrum has not been observed. Chromocenium ion exhibits a ${ }^{4} \mathrm{~A}_{2 \mathrm{~g}}$ $\mathrm{d}^{3}\left(\delta^{2} \sigma^{1} \pi^{0}\right)$ ground state. A theoretical estimate of $D$ for chromocenium ion may be obtained from the transition energies of the spin-allowed transitions of the chromocenium ion. These were taken from the spectrum of cobaltocenium iodide in aqueous solution [30] and used to compute the Racah $B$ parameter $\left(513 \mathrm{~cm}^{-1}\right)$. The value of the $B$ is needed to calculate the positions of two low-lying ${ }^{2} \mathrm{E}_{\mathrm{lg}}\left(\delta^{1} \sigma^{2} \pi^{0}\right)$ states that make large spin-orbit coupling contributions to $D$. The spin-orbit contribution to the zerofield splitting was then computed from second-order perturbation theory. We obtain a value of $5.88 \mathrm{~cm}^{-1}$ for the spin-orbit contribution to $D$ and this will be increased slightly by addition of the spin-spin contribution. The values of $g_{\|}$and $g_{\perp}$ were also obtained in this calculation as 1.979 and 2.0023 , respectively.

The spin-Hamiltonian for chromocenium is given in eq. (1), with $S=3 / 2$. For sufficiently large values of $D\left(>0.5 \mathrm{~cm}^{-1}\right)$ the only observable transitions in an $X$-band spectrometer are those of the spin-1/2 Kramers doublet and the resonance fields for $E=0$ are given by:

$H_{\mathrm{r}}=\frac{g_{\mathrm{c}} \beta H_{\mathrm{c}}}{\sqrt{g_{\|}^{2} \cos ^{2} \theta+4 g_{\perp}^{2} \sin ^{2} \theta}}$.

In eq. (7), $H_{\mathrm{e}}$ is the spectrometer resonance field for a free electron $(3320 \mathrm{G}), g_{\|}$and $g_{\perp}$ are expected to be close to $g_{\mathrm{e}}$. Therefore, when $\boldsymbol{H} \| \mathrm{C}_{5}$, eq. (7) predicts a resonance field $H_{\mathrm{r}}=3333 \mathrm{G}$, and when $\boldsymbol{H} \perp \mathrm{C}_{5}$, 
$H_{\mathrm{r}}=1670 \mathrm{G}$. Since an ESR signal is observed, some or all of the chromocene is oxidized upon intercalation. The ESR spectrum is consistent with orientation of the chromocenium $\mathrm{C}_{5} \perp C^{*}$. In this case the $\boldsymbol{H} \| \boldsymbol{C}^{*}$ spectrum should consist of a single, symmetrical resonance at about $H_{\mathrm{e}} / 2=1670 \mathrm{G}$ as is, in fact, observed.

The resonance fields of the satellite bands near $g_{\perp}$ vary somewhat from one crystal to another. The absence of any high-field transition below $10 \mathrm{kG}$ indicates that $D>0.5 \mathrm{~cm}^{-1}$. For $D>0.5 \mathrm{~cm}^{-1}$, the position of the $g_{\|}$resonance is insensitive to the value of $E$. However, the position of the $g_{\perp}$ resonance is shifted for $E \neq 0$. Thus, it appears that the satellite structure is due to sites with $E$ values differing from zero by about $0.1 \mathrm{~cm}^{-1}$.

The $\boldsymbol{H} \| \boldsymbol{C}^{*}$ spectrum was computed by diagonalization of eq. (1) with $D=5.88 \mathrm{~cm}^{-1}$ and $E=0$. The satellite feature was reproduced with $D=5.88 \mathrm{~cm}^{-1}$ and $E=0.2 \mathrm{~cm}^{-1}$. The computed spectrum is illustrated in fig. 5. Of course, the site symmetry of the chromocenium ion in the VWG does not permit rigorous axial symmetry, but the axial field of the ligands is evidently far stronger than the crystal field $(|D| \gg|E|)$.

A two-dimensional "powder" spectrum should be observed with $\boldsymbol{H} \perp \boldsymbol{C}^{*}$. The spectrum was calculated by averaging eq. ( 1 ) over all values of $\theta$ to obtain:

$$
\begin{aligned}
& I(H) \propto \\
& \frac{\left(g_{\mathrm{e}} H_{\mathrm{e}}\right)^{2}+\left(g_{\|} H_{\mathrm{e}}\right)^{2}}{H\left\{\left[\left(g_{\mathrm{e}} H_{\mathrm{e}}\right)^{2}-\left(g_{\|} H\right)^{2}\right]\left[\left(g_{\perp} H\right)^{2}-\left(g_{\mathrm{e}} H\right)^{2}\right]\right\}^{1 / 2}} .
\end{aligned}
$$

Eq. (8) has significant amplitude at $g_{\|}$and $g_{\perp}$. However, the $\boldsymbol{H} \perp \boldsymbol{C}^{*}$ spectrum could not be observed due to its weakness and interference from trace paramagnetic impurities with $g=2$.

The failure to observe $\mathrm{Cr}(\mathrm{cp})_{2}^{+}$resonance at room temperature is consistent with the rapid reorientational motion of the ion at higher temperatures as suggested by neutron scattering studies [20]. We assume that thermal motion leads to a rapid spin-lattice relaxation and associated linebroadening. A similar temperature dependence observed for $\mathrm{Co}(\mathrm{cp})_{2}$ may be attributed to the presence of lowlying electronic excited states.

\section{Conclusion}

None of the intercalated metallocenes examined in the present study can be detected by ESR spectroscopy in both the neutral and cationic form. Therefore, it has not been possible to establish the simultaneous presence of multiple oxidation states in the VWG. It has been possible to demonstrate the presence of oxidized chromocene and nickelocene generated from the neutral metallocene intercalate. Cobaltocene was shown to be present in unoxidized form. The results imply that the metallocene intercalation compounds of $\mathrm{M}_{2} \mathrm{P}_{2} \mathrm{X}_{6}$ lattices contain mixtures of both neutral and oxidized species. This implication is consistent with the results of recent experiments with cobaltocene intercalated $\mathrm{SnS}_{2}$ [28]. $\mathrm{X}$-ray photoelectron spectroscopy revealed the presence of $\mathrm{Co}^{3+}, \mathrm{Co}^{2+}, \mathrm{Sn}^{4+}$ and probably $\mathrm{Sn}^{2+}$ in the intercalated lattice. The ESR spectrum of a powdered sample of $\mathrm{SnS}_{2}\left[\mathrm{Co}(\mathrm{cp})_{2}\right]_{0.31}$ was analyzed in terms of an axially symmetric $g$-tensor with $g_{\perp}=2.092$ and $g_{\|}=1.96$; close to the values observed for cobaltocene intercalated $\mathrm{Cd}_{2} \mathrm{P}_{2} \mathrm{~S}_{6}$. Similarly, ferrocene intercalated $\mathrm{Cd}_{2} \mathrm{P}_{2} \mathrm{~S}_{6}$ has been shown by Mössbauer spectroscopy to contain a range of oxidation states of iron [19].

The metallocene orientation within the VWG could be determined for the paramagnetic species. Both cobaltocene and nickelocene cation are oriented with $\mathrm{C}_{5}$ parallel to the $C^{*}$ stacking axis. Chromocene cation is oriented with $C_{5}$ perpendicular to $C^{*}$. $\mathrm{Co}(\mathrm{cp})_{2}$ and $\mathrm{Ni}(\mathrm{cp})_{2}^{+}$are isoelectronic $\left(\delta^{4} \sigma^{2} \pi^{1}\right)$ with a single electron in the strongly antibonding $\pi\left(\mathrm{d}_{x z}, \mathrm{~d}_{y z}\right)$ orbital; the $\pi$-orbital of $\mathrm{Cr}(\mathrm{cp})_{2}$ is unoccupied. These observations suggest that back-bonding from lattice sulfur orbitals into the vacant metallocene $d_{x z}, d_{y z}$ orbitals may play a role in establishing the $\mathrm{C}_{5} \| C^{*}$ orientation.

No additional ESR resonances were observed that could be attributed to free electrons transferred from nickelocene or chromocene to the host lattice. The location of the electrons donated to the lattice has been a longstanding problem. It has been anticipated that electrons donated to the lattice could occupy states in the narrow, phosphorus-phosphorus $\sigma$-antibonding band expected to be the lowest energy unoccupied band of the solid. Some evidence has been presented both supporting and challenging this sup- 
position [29,30]. Recently, band calculations have suggested another possibility: that the electrons are donated to unoccupied $d$ bands [31]. However, in $\mathrm{Cd}_{2} \mathrm{P}_{2} \mathrm{~S}_{6}\left(\mathrm{~d}^{10}\right)$, the $\mathrm{d}$ bands are fully occupied and not unavailable for charge donation.

\section{References}

[1] V.W. Klingen, G. Fulenberger and H. Hahn, 7. Anorg. Allgem. Chem. 401 (1973) 97.

[2] B.E. Taylor, J. Steger and A. Wold, J. Solid State Chem. 7 (1973) 461 .

[3] M.B. Dines, Science 188 (1975) 1210.

[4] R.P. Clement, W.B. Davies, K.A. Ford, M.L.H. Green and A.J. Jacobson, Inorg. Chem. 17 (1978) 2754.

[5] B.G. Silbernagel, Chem. Phys. Letters 34 (1975) 298.

[6] F.R. Gamble and A.H. Thompson, Solid State Commun. 27 (1978) 379.

[7] F.R. Gamble and T.H. Geballe, Treatise Solid State Chem. 3 (1976) 89.

[8] H.D. Zagefka and R. Schollhorn, Angew. Chem. Intern. Ed. 16 (1977) 199.

[9] R. Clement, W. Davies, K. Ford, M. Green and A.J. Jacobson, J. Mol. Catal. 3 (1977) 191.

[10] M.B. Dines and P.M. DiGiacomo, Inorg. Chem. 20 (1981) 92.

[11] J.W. Johnson, in: Intercalation chemistry, eds. M.S. Whittingham and A.J. Jacobson (Academic Press, New York, 1982) p. 267.

[12] Y. Mathey, R. Clement, C. Sourisseau and G. Lucazeau, Inorg. Chem. 19 (1980) 2773.

[13] D. Cleary and A.H. Francis, J. Phys. Chem. 89 (1985) 97.
[14] J.P. Audiere, R. Clement, Y. Mathcy and C. Mazicres, Physica B + C 99 (1980) 133.

[15] R. Clement, O, Garnier and Y. Mathey, Nouv. J. Chim. 6 (1982) 13.

[16] R. Clement, J. Girerd and I. Morgenstern-Badarau, Inorg. Chem. 19 (1980) 2852.

[17] C. Sourisseau, J.P. Forgerit and Y. Mathey, J. Phys. Chem. Solids 44 ( 1983 ) 119.

[18] Y. Mathey, R. Clement, C. Sourisseau and G. Lucazeau, Inorg. Chem. 19 (1980) 2773.

[19] B. Bal, S. Ganguli and M. Bhattacharya, Physica B 133 (1985) 64.

[20] C. Sourisseau, Y. Mathey and C. Poinsignon, Chem. Phys. 71 (1982) 257.

[21] R. Clement, J. Chem. Soc. Chem. Commun. 19 (1980) 647.

[22] R. Clement. O. Garnier, H. Mercier, J.-P. Audiere, A. Michalowicz, B. Rousseau and R. Setton, J. Chem. Soc. Chem. Commun. (1984) 1354.

[23] A. Michalowicz, G. Vlaic, R. Clement and Y. Mathey, Springer Ser. Chem. Phys. 27 (1983) 222.

[24] B.E. Taylor, J. Steger and A. Wold, J. Solid State Chem. 7 (1973) 461 .

[25] J.H. Ammeter, J. Magn. Reson. 30 (1978) 299.

[26] K.D. Warren, Struct. Bonding 27 (1976) 45.

[27] J.H. Ammeter Jr. and J.D. Swalen, J. Chem. Phys. 57 (1972) 678.

[28] D. O'Hare, W. Jaegermann, D.L. Williamson, F.S. Ohuchi and B.A. Parkinson, Inorg. Chem. 27 (1988) 1537.

[29] C. Berthier, Y. Chabre and M. Minier, Solid State Commun. 28 (1978) 327.

[30] M.S. Whittingham, in: Solid state electrolytes, eds. P. Hagenmuller and W. van Gool (Academic Press, New York, $1978)$ p. 375.

[31] M.-H. Whangbo, J. Rouxel and L. Trichet, Inorg. Chem. 24 (1985) 1824. 